Research Paper

\title{
Dynamic Differences Of Red Cell Distribution Width Levels Contribute To The Differential Diagnosis Of Hepatitis B Virus-related Chronic Liver Diseases: A Case-control Study
}

\author{
Mengjie Zhu, Man Han, Xiaoyu Xiao, Songsong Lu , Zhao Guan, Ying Song, Chen Liu ${ }^{\bowtie}$ \\ Department of Clinical Laboratory, Peking University People's Hospital, Beijing, China \\ $\triangle$ Corresponding author: Chen Liu, PhD. Department of Clinical Laboratory, Peking University People's Hospital. 11\# Xizhimen South Street, Beijing, China. \\ Phone/Fax: +8610 88326203. E-mail address: liuchen-best@pku.edu.cn. \\ (C) Ivyspring International Publisher. This is an open access article distributed under the terms of the Creative Commons Attribution (CC BY-NC) license \\ (https://creativecommons.org/licenses/by-nc/4.0/). See http://ivyspring.com/terms for full terms and conditions.
}

Received: 2018.11.27; Accepted: 2019.03.27; Published: 2019.05.10

\begin{abstract}
Objective: This study aims to clarify the changes and clinical significance of red cell distribution width (RDW) during HBV-related chronic diseases, including inactive hepatitis B virus (HBV) carriers, HBV immune tolerant individuals, chronic hepatitis $B(\mathrm{CHB})$ patients and HBV-related hepatocirrhosis patients.

Methods: RDW was measured $288 \mathrm{CHB}$ patients, 100 patients with hepatitis B e antigen( $\mathrm{HBeAg}$ )-negative chronic $\mathrm{HBV}$ infection (inactive carriers), 92 patients with $\mathrm{HBeAg}$-positive chronic HBV infection (immune tolerant), and 272 patients with HBV-related hepatocirrhosis. Their RDW changes were compared with 160 healthy controls. Correlations between RDW and clinical indicators were conducted. For $\mathrm{HBeAg}+\mathrm{CHB}$ patients, RDW was measured before and after antiviral therapy. The efficiency of RDW to distinguish hepatocirrhosis from $\mathrm{CHB}$ and/or inactive carriers was evaluated by receiver operating characteristic (ROC) curves.

Results: RDW was higher in hepatocirrhosis patients than other groups of patients and healthy controls. Besides, $\mathrm{HBeAg}+\mathrm{CHB}$ patients possessed higher RDW than $\mathrm{HBeAg}-\mathrm{CHB}$ patients. For $\mathrm{HBeAg}+$ patients that underwent $\mathrm{HBeAg}$ seroconversion after antiviral therapy, RDW was decreased. RDW was positively correlated with total bilirubin and Child-Pugh scores and negatively correlated with albumin among hepatocirrhosis patients. The areas under the curve (AUC) of ROC curves to distinguish hepatocirrhosis from CHB patients was 0.7040 for RDW-standard deviation (RDW-SD) and 0.6650 for RDW-coefficient of variation (RDW-CV), and AUC to distinguish hepatocirrhosis from inactive carriers was 0.7805 for RDW-SD and 0.7991 for RDW-CV.

Conclusions: RDW is significantly increased in $\mathrm{HBeAg}+\mathrm{CHB}$ patients and patients with HBV-related hepatocirrhosis and could reflect their severity. RDW could help to distinguish hepatocirrhosis from CHB patients and inactive HBV carriers.
\end{abstract}

Key words: hepatocirrhosis; chronic hepatitis B; Red blood cells; Red cell distribution width.

\section{Introduction}

Red blood cell distribution width (RDW) is one hematological indicator which measures size variability of circulating erythrocytes and reflects the degree of heterogeneity of erythrocyte volume. RDW is routinely used in laboratory hematology for differential diagnosis of anemias [1-2]. Nonetheless, the assessment of this parameter is broadened far beyond the differential diagnosis of anemias. RDW had also emerged as a prognostic marker in a variety of disorders such as cardiovascular disease, cancer, 
diabetes, chronic obstructive pulmonary disease, kidney failure, as well as in other acute or chronic conditions. The increase of RDW is of high predictive value for diagnosing a variety of disorders [3-10].

Worldwide, hepatitis B virus (HBV) infection is a major health problem and chronic infection with hepatitis B virus (HBV) is estimated to affect 350 million people in the world [11]. In China, where HBV infection is endemic, there are estimated 93 million HBV carriers, and among them 30 million are patients with chronic hepatitis B [12]. Chronic HBV infection is associated with a wide range of clinical manifestations, from an asymptomatic carrier status with normal liver histology to chronic liver diseases [12-13]. According to EASL 2017 Clinical Practice Guidelines on the management of hepatitis B virus infection, the natural history of chronic HBV infection has been schematically divided into five phases: HBeAg-positive chronic HBV infection (immune tolerant), HBeAg-positive chronic hepatitis B, HBeAg-negative chronic HBV infection (inactive carrier), HBeAg-negative chronic hepatitis $\mathrm{B}$ and HBsAg-negative phase [14]. In addition, about $15 \%$ to $40 \%$ of chronically infected people may develop to chronic and progressive liver diseases, including cirrhosis and hepatocellular carcinoma (HCC), whereas the remainders become inactive carriers [15]. The incidence rates of cirrhosis in chronic HBV infection range from $2 \%$ to $7 \%$ annually and meanwhile the cirrhosis incidence rate was $0.7 \%$ among asymptomatic HBV carriers annually [16-18], so that distinguishing cirrhosis patients from $\mathrm{CHB}$ patients and $\mathrm{HBV}$ carriers is quite meaningful.

Concerning about the significance of RDW in HBV-related diseases, some researches had been reported [19-24]. RDW values were reported to be significantly increased in patients with hepatitis $B$ and were associated with its severity [19-20]. RDW can be defined as independent predicting factors in hepatic fibrosis and necroinflammation [21-22]. RDW to Platelet Ratio (RPR) was reported to be able to predict fibrosis and cirrhosis in CHB patients [23]. However, few researches concern about the dynamic differences of RDW levels during HBV-related chronic liver diseases and the knowledge of clinical significance of RDW is also limited. Fan $X$ and his colleagues reported that RDW among CHB patients was elevated compared with healthy controls, based on meta-analysis [24].

This study aims to provide general information about dynamic differences of RDW levels among hepatitis B virus-related chronic liver diseases. We compared the changes of RDW among chronic HBV infection (inactive carrier and immune tolerant), chronic hepatitis $\mathrm{B}$, and HBV related hepatocirrhosis patients, we also analyzed whether RDW levels were different between HBeAg-positive and negative CHB patients, and before and after anti-virus treatment. We inquired into the significance of RDW in HBV-related chronic diseases and gave some insights about its potential clinical applications.

\section{Materials and Methods}

\section{Patients}

Chinese subjects with related disease diagnosis were retrospectively enrolled in this study from our Hospital from March 2014 to Oct 2017. Based on EASL 2017 Clinical Practice Guidelines on the management of hepatitis B virus infection, the natural history of chronic HBV infection has been schematically divided into five phases: HBeAg-positive chronic $\mathrm{HBV}$ infection (immune tolerant), HBeAg-positive chronic hepatitis $\mathrm{B}, \mathrm{HBeAg}$-negative chronic HBV infection (inactive carrier), $\mathrm{HBeAg}$-negative chronic hepatitis $\mathrm{B}$ and HBsAg-negative phase [14]. Accordingly, we included 92 patients with HBeAg-positive chronic HBV infection (immune tolerant), 100 patients with HBeAg-negative chronic $\mathrm{HBV}$ infection (inactive carriers), and one case group of 288 chronic hepatitis B patients(156HBeAg- and $\left.132 \mathrm{HBeAg}^{+}\right)$, all these patients were recruited according to standards in EASL 2017 Clinical Practice Guidelines on the management of hepatitis B virus infection[14]. Another case group included 272 patients with HBV-related hepatocirrhosis, according to the following criteria: hepatitis B surface antigen (HBsAg) carrier for $\geq 6$ months; pathological or clinical evidence of cirrhosis, including nodularity/splenomegaly on liver imaging and/or thrombocytopenia. Child-Pugh score for each hepatocirrhosis patient was calculated as previously reported [25]. Patients with HCC, a history of other malignancy, other forms of irrelevant liver diseases, including hepatitis $C$ virus, serious alcoholic disease, or autoimmune hepatitis, or patients who had experienced blood transfusions within six months were excluded. Among the HBeAg+ CHB patients, 65 patients who performed entecavir treatment for more than half a year (6 to 9 months) were analyzed, and changes in RDW before and after antiviral therapy were compared. The control group included 160 healthy adults, who had taken physical examination in the hospital. All selected individuals were without other irrelevant chronic diseases and anybody with anemia was also excluded. All the procedures were carried out in accordance with Helsinki Declaration, with approval from institutional ethical review board of Peking University People's Hospital. 


\section{Clinical parameter analysis}

Whole blood specimens were obtained by venipuncture into vacuette tubes containing K2-EDTA(BD, Franklin Lakes, NJ, USA) and immediately analyzed to obtain hematological variables using an automated hematology analyzer (Sysmex XN-9000; Sysmex, Kobe, Japan). RDW-SD and $\mathrm{RDW}-\mathrm{CV}$ were both measured and calculated. $\mathrm{RDW}-\mathrm{CV}$ is equal to RDW-SD/MCV. HBsAg and $\mathrm{HBeAg}$ were measured by chemiluminescent micro-particle immunoassay (CMIA) (Architect i2000 SR, Abbott, IL, USA). Real time PCRs for quantification of HBV DNA were carried out in the LightCycler 480 instrument (Roche Applied Sciences). The levels of aspartate aminotransferase (AST), alanine aminotransferase (ALT), total bilirubin and albumin in serum were measured by Beckman Coulter AU5800 automatic biochemical analyzer (Beckman Coulter Inc., Brea, CA, USA). $\mathrm{HBsAg}<0.05 \mathrm{IU} / \mathrm{ml}$, $\mathrm{HBsAb}<10 \mathrm{~S} / \mathrm{CO}$, $\mathrm{HBeAg}<1 \mathrm{~S} / \mathrm{CO}, \mathrm{HBeAb}<1 \mathrm{~S} / \mathrm{CO}, \mathrm{HBcAb}<1 \mathrm{~S} / \mathrm{CO}$, $\mathrm{HBV}$ DNA $<1000 \mathrm{IU} / \mathrm{ml}, \mathrm{ALT}<40 \mathrm{U} / \mathrm{L}, \mathrm{AST}<35 \mathrm{U} / \mathrm{L}$ were regarded as negative, according to the manufacturer's instructions.

\section{Statistics}

Analyses were carried out with GraphPad Prime 5.5 and SPSS software. ANOVA with the Bonferroni's Multiple Comparison Test were used to compare RDW of different groups. RDW levels before and after therapies were compared by paired student's $t$ test. The Pearson correlation coefficient $(r)$ was estimated between RDW and clinical indicators and Spearman correlation coefficient (r) was estimated between RDW and Child-Pugh scores. Receiver operating characteristics (ROC) curves were used to evaluate the usefulness of RDW to distinguish hepatocirrhosis from $\mathrm{CHB}$ or inactive carriers and area under curves (AUC) was calculated and optimal cut-off values were analyzed according to the maximum value of Youden index (Youden index=sensibility +specificity -1). All of the statistical tests were 2-tailed. P values less than
0.05 are regarded as significant.

\section{Results}

\section{Increased RDW in patients with CHB or HBV-related hepatocirrhosis}

The clinical and demographic characteristics of individuals involved in this study were summarized in Table 1. There was no significant difference in the percentage of gender and age distribution $(\mathrm{P}>0.05)$ among different groups. For hematological indicators, however, the number of white blood cells and platelets were significant lower in HBV-related hepatocirrhosis patients than other groups $(p<0.05)$, which is in accordance with previous studies [26-28].

The levels of RDW, both coefficient of variation (CV) and standard deviation (SD), of each group were analyzed and compared by ANOVA with multiple comparison and the results are shown in Figure 1. We found that $\mathrm{RDW} \mathrm{CV}$ and $\mathrm{SD}$ were generally significantly higher in $\mathrm{CHB}$ patients and patients with HBV-related hepatocirrhosis $(\mathrm{P}<0.05)$ than in healthy controls, inactive HBV carriers and immune tolerant individuals, with the exception that RDW-SD levels were not significantly different between $\mathrm{CHB}$ patients and inactive carriers. Patients with HBV-related hepatocirrhosis had significant higher RDW than CHB patients. Levels of RDW were not different among healthy controls, inactive HBV carriers and immune tolerant individuals.

\section{Higher RDW in HBeAg positive CHB patients than $\mathrm{HBeAg}$ negative $\mathrm{CHB}$ patients}

We further divided $\mathrm{CHB}$ patients into $\mathrm{HBeAg}+$ and $\mathrm{HBeAg}$ - subgroups since it represents different stages of CHB [14, 29]. The demographic and clinical characters of the subgroups were shown in Table S1. We compared RDW values to decide whether they were different (Figure 2). We found that the levels of $\mathrm{RDW}$ were significant higher in $\mathrm{HBeAg}+\mathrm{CHB}$ patients than $\mathrm{HBeAg}+\mathrm{CHB}$ patients and healthy controls, for both RDW-SD and RDW-CV.

Table 1. Demographic and clinical characters of the subjects in the study

\begin{tabular}{|c|c|c|c|c|c|}
\hline & Healthy controls & immune tolerant & $\mathrm{CHB}$ & Inactive carriers & Hepatocirrhosis \\
\hline $\mathrm{N}$ & 160 & 92 & 288 & 100 & 272 \\
\hline gender & $79 \mathrm{~F} / 81 \mathrm{M}$ & $45 \mathrm{~F} / 47 \mathrm{M}$ & 144F/144M & $49 \mathrm{~F} / 51 \mathrm{M}$ & 135F/137M \\
\hline Age(year) & $44.73 \pm 15.45$ & $41.23 \pm 14.01$ & $40.17 \pm 11.71$ & $40.56 \pm 11.62$ & $41.20 \pm 10.60$ \\
\hline $\operatorname{RBC}\left(\times 10^{12} / \mathrm{L}\right)$ & $4.80 \pm 0.98$ & $5.12 \pm 1.51$ & $5.04 \pm 1.06$ & $5.24 \pm 0.61$ & $4.48 \pm 0.89$ \\
\hline $\mathrm{WBC}\left(\times 10^{9} / \mathrm{L}\right)$ & $6.57 \pm 1.73$ & $6.09 \pm 2.09$ & $5.38 \pm 1.72 *$ & $6.22 \pm 1.86$ & $4.72 \pm 1.95^{* * *}$ \\
\hline Lymphocyte (\%) & $34.44 \pm 7.39$ & $354.94 \pm 9.35$ & $34.96 \pm 8.38$ & $36.35 \pm 9.40$ & $32.75 \pm 9.54$ \\
\hline Lymphocyte $\left(\times 10^{9} / \mathrm{L}\right)$ & $2.24 \pm 0.70$ & $2.04 \pm 0.74$ & $1.82 \pm 0.59 *$ & $2.20 \pm 0.73$ & $1.51 \pm 0.63^{* * *}$ \\
\hline Neutrophil (\%) & $56.37 \pm 7.79$ & $53.21 \pm 10.12$ & $53.62 \pm 9.26$ & $54.27 \pm 10.01$ & $55.23 \pm 10.30$ \\
\hline Neutrophil $\left(\times 10^{9} / \mathrm{L}\right)$ & $3.75 \pm 1.28$ & $3.37 \pm 1.76$ & $2.95 \pm 1.22$ & $3.44 \pm 1.42$ & $2.67 \pm 1.47^{* * *}$ \\
\hline $\operatorname{PLT}\left(\times 10^{9} / \mathrm{L}\right)$ & $227.8 \pm 50.8$ & $219.2 \pm 86.9$ & $196.6 \pm 73.6$ & $207.8 \pm 66.3$ & $120.4 \pm 62.2^{* * *}$ \\
\hline
\end{tabular}

*** $\mathrm{p}<0.001,{ }^{*} \mathrm{p}<0.05$, compared with healthy controls 

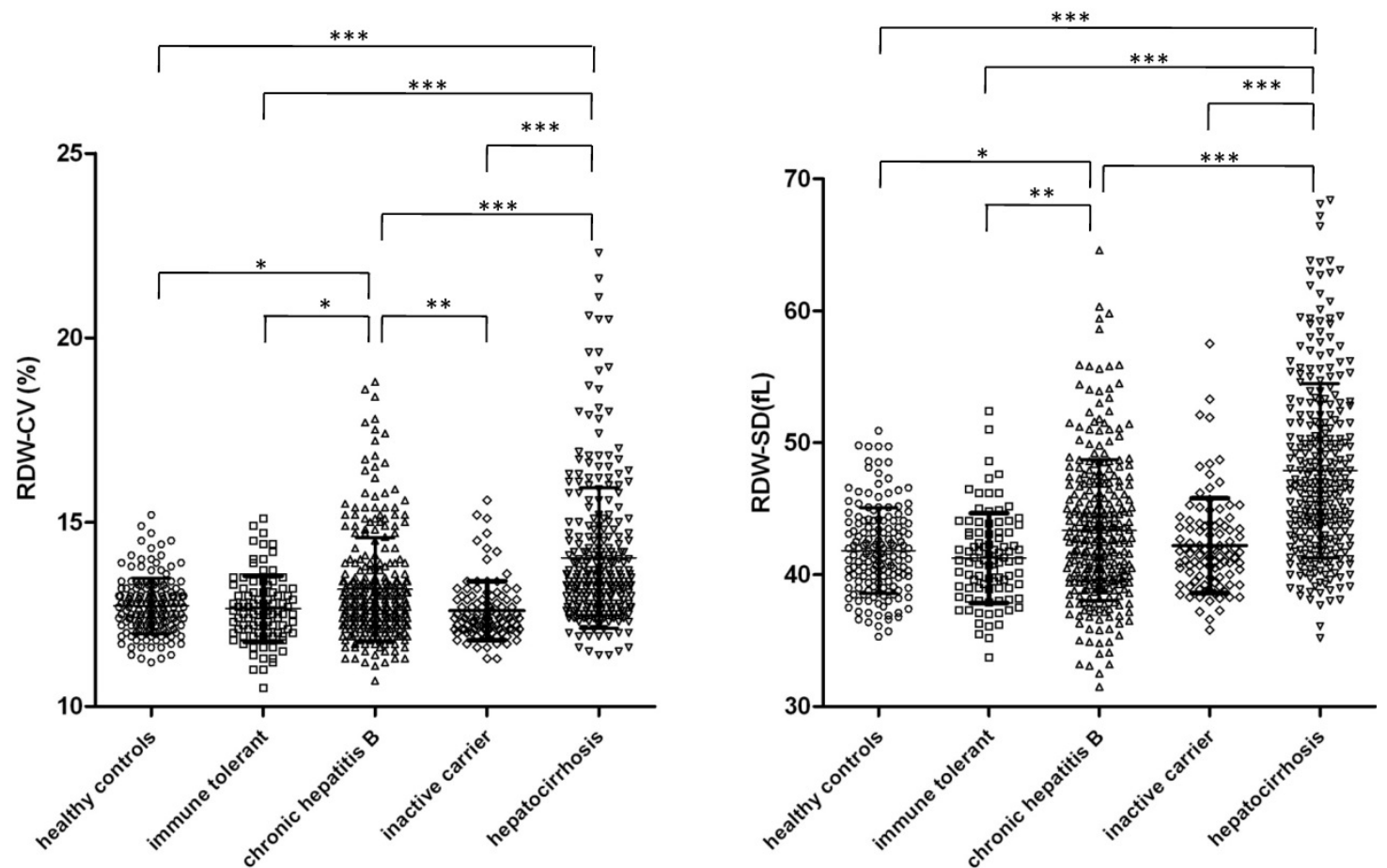

Figure 1. Levels of RDW in inactive HBV carriers, immune tolerant individuals, chronic hepatitis $B$ patients and $\mathrm{HBV}$ related hepatocirrhosis patients. CV (left) and SD (right) of RDW in asymptomatic HBV carriers $(n=100)$, immune tolerant individuals $(n=92)$, chronic hepatitis $B$ patients $(n=288)$, HBV related hepatocirrhosis patients $(n=272)$ and healthy controls $(n=160)$ were analyzed and compared with each other. Data points are shown with means \pm standard deviations and ANOVA with the Bonferroni's Multiple Comparison Test were used to compare RDW of different groups. *** $p<0.001 ;{ }^{* *} p<0.01 ;{ }^{*} p<0.05$.
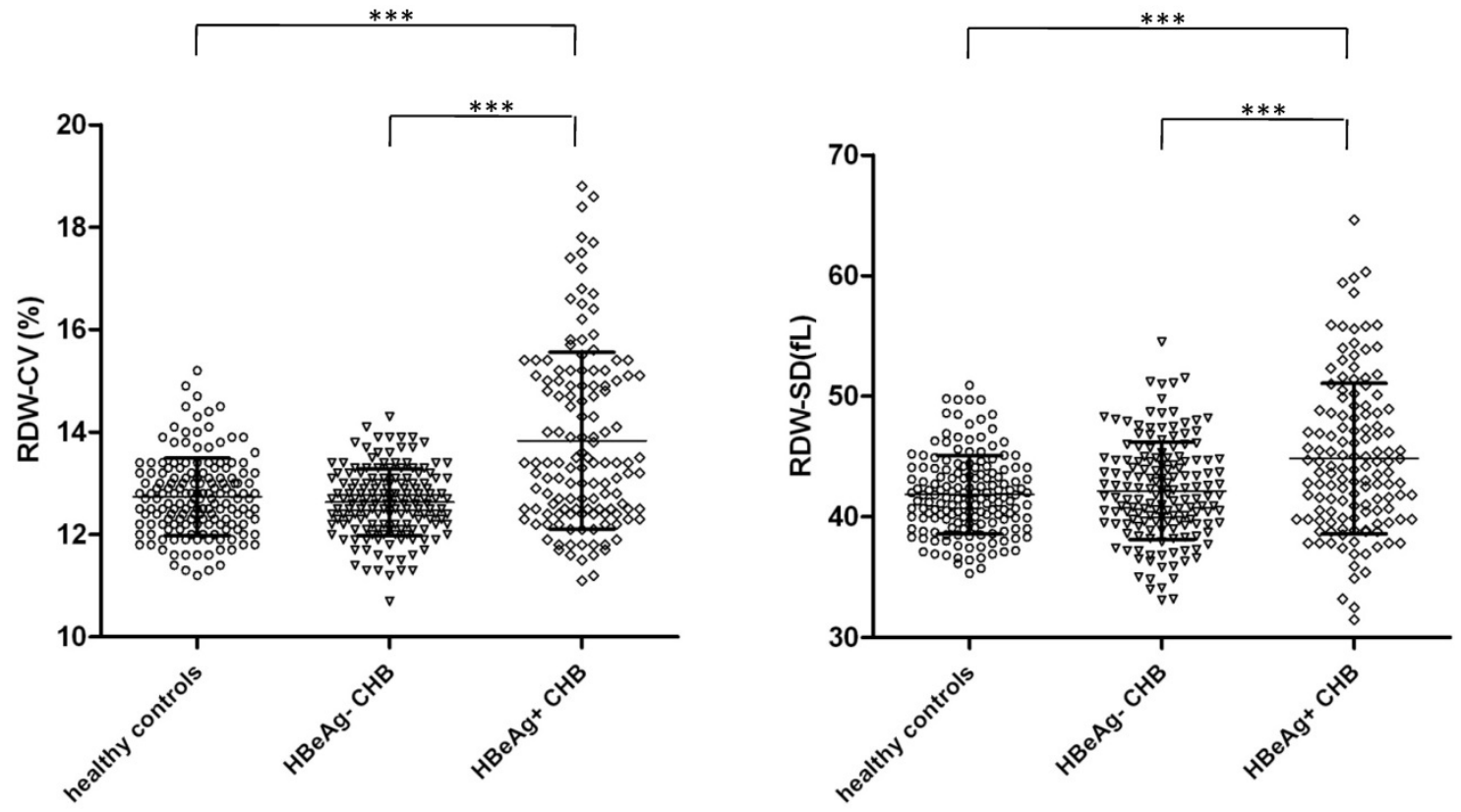

Figure 2. RDW levels in subgroups of CHB patients according to HBeAg expression. The levels of RDW, both CV (left) and SD (right) in HBeAg- CHB patients $(n=156)$ and $\mathrm{HBeAg}+\mathrm{CHB}$ patients $(n=132)$ were analyzed and compared with each other as well as with healthy controls $(n=160)$. Data points are shown with means \pm standard deviations and ANOVA with the Bonferroni's Multiple Comparison Test were used to compare RDW of different groups. **** $<<0.001$.

\section{Correlations between clinical indicators and RDW were weak for $\mathrm{CHB}$ patients and inactive HBV carriers.}

We next inquired into the relationship between RDW and clinical indicators to clarify the clinical significance of RDW. First we analyzed the role of RDW in CHB patients, asymptomatic HBV carriers and immune tolerant individuals. Correlations were conducted between RDW (both $\mathrm{CV}$ and SD) and clinical indicators, including $\mathrm{HBsAg}, \mathrm{HBeAg}, \mathrm{HBeAb}$, 
HBV-DNA, ALT and AST, and the results are shown in Table S2. We found that though some correlations were with $\mathrm{p}$ values less than 0.05 , the correlation coefficients (r) were too low, suggesting that the correlations were weak. We also further analyzed correlations in sub-groups of $\mathrm{CHB}$ patients according to HBeAg, but these were also no significant correlations (data not shown).

\section{RDW was decreased after antiviral therapy for $\mathrm{HBeAg}+\mathrm{CHB}$ patients}

Among the $132 \mathrm{HBeAg}+\mathrm{CHB}$ patients, 65 patients were treated with entecavir for more than half a year, and changes in RDW before and after antiviral therapy were compared and analyzed. Among the 65 patients, 47 were diagnosed to become $\mathrm{HBeAg}$ - after treatment and 18 were still $\mathrm{HBeAg}$. We separately compared RDW levels before and after treatment for these two groups, as shown in Figure 3. For patients that were still $\mathrm{HBeAg}+$ after treatment, RDW-SD and RDW-CV were not significantly changed before and after treatment. For patients that underwent HBeAg seroconversion, RDW-SD and RDW-CV were both significantly decreased after antiviral therapy. These results suggest that RDW level changes are associated with the outcome of disease in patients with $\mathrm{CHB}$ after therapy.

\section{RDW was significantly correlated with total bilirubin, albumin and Child-Pugh scores of patients of with HBV-related hepatocirrhosis.}

We next analyzed the relationship of RDW with clinical indicators among patients with HBV-related hepatocirrhosis. Clinical indicators that reflect severity of hepatocirrhosis were collected and their correlations with RDW were carried out. We found that there were significant positive correlations between RDW-CV and T-BIL, between RDW-SD and T-BIL. Besides, there were also significant negative correlations between RDW (both $\mathrm{CV}$ and SD) and albumin (Figure 4). In addition, RDW-SD and CV were also positively correlated with AST (not shown). As reported, T-BIL is elevated and albumin is decreased in serum of hepatocirrhosis patients [30], and our results suggested that increased RDW in HBV-related hepatocirrhosis was related to the severity of the disease.
RDW-CV
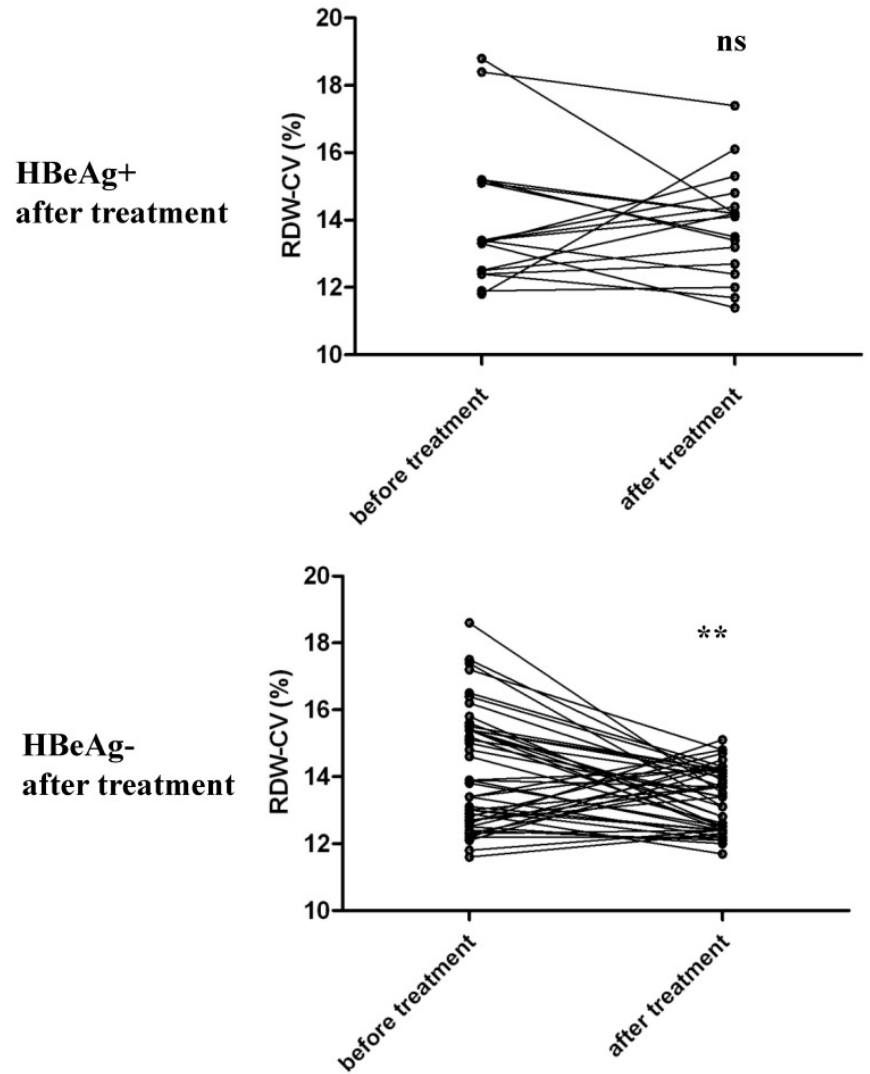
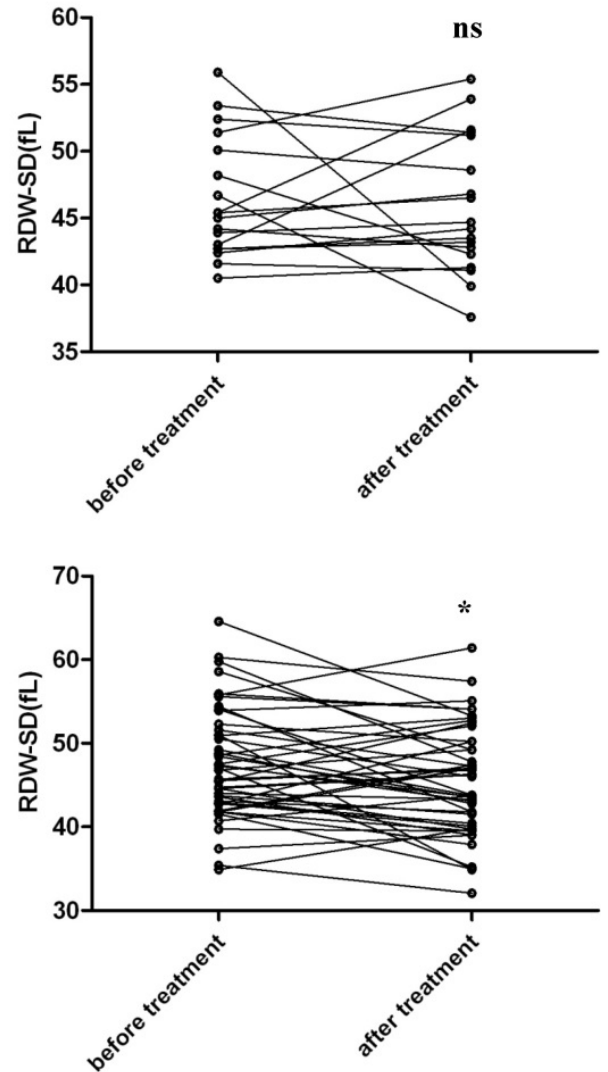

Figure 3. Comparison of RDW levels of HBeAg+ CHB patients before and after antiviral therapy. $65 \mathrm{HBeAg}+\mathrm{CHB}$ patients who were treated with entecavir for more than half a year were enrolled and RDW levels before and after treatment were analyzed. Among the 65 patients, 47 were diagnosed to become $\mathrm{HBeAg}$ - after the treatment and 18 were still $\mathrm{HBeAg+}$. We separately compared RDW levels before and after treatment for these two groups for both RDW-CV and RDW-SD, by paired student's t test. *** $\mathrm{p}<0.001$; * $\mathrm{p}<0.05$; ns, not significant. 
We next calculated Child-Pugh scores for each hepatocirrhosis patients and correlation analysis was conducted between RDW and Child-Pugh scores, as shown in Figure 4, there were significant positive correlations between RDW (both $\mathrm{CV}$ and SD) and Child-Pugh scores, which prove that RDW is associated with the severity of HBV-related hepatocirrhosis.

\section{RDW is of usefulness to distinguish HBV-related hepatocirrhosis patients from CHB patients and inactive HBV carriers.}

The diagnosis of hepatocirrhosis among CHB patients and inactive HBV carriers was mainly dependent on liver imaging and a series of clinical tests. Our results suggested that patients with HBV-related hepatocirrhosis were with significantly higher RDW than $\mathrm{CHB}$ patients and inactive HBV carriers, suggesting the potential diagnosis value of RDW to distinguish HBV-related hepatocirrhosis patients from $\mathrm{CHB}$ and $\mathrm{HBV}$ carriers. We did preliminary receiver operating characteristics (ROC) curve analysis to evaluate the usefulness of RDW and area under curves (AUC) was calculated. As shown in Figure 5, we found that The AUC of ROC curves to distinguish hepatocirrhosis from $\mathrm{CHB}$ patients was 0.7040 for RDW-SD and 0.6650 for RDW-CV, and AUC to distinguish hepatocirrhosis from inactive HBV carriers was 0.7805 for RDW-SD and 0.7991 for $\mathrm{RDW}-\mathrm{CV}$. If we put inactive carriers and $\mathrm{CHB}$ patients together as the control group for ROC analysis, the AUC was 0.7237 for RDW-SD and 0.6995 for RDW-CV. The optimal cut-off values for RDW (CV and SD) in each situation were calculated from ROC curves by the maximum values of Youden index, as shown in Table 2. We also regarded immune tolerant individuals or immune tolerant individuals together with inactive carriers and $\mathrm{CHB}$ patients as the control group and calculated AUC (Table S3), and the AUC were 0.8187 and 0.7419 for RDW-SD, 0.7597 and 0.7111 for RDW-CV. These results suggested that RDW could be used as one potential indicator that helps to diagnose hepatocirrhosis from other HBV-related chronic diseases.
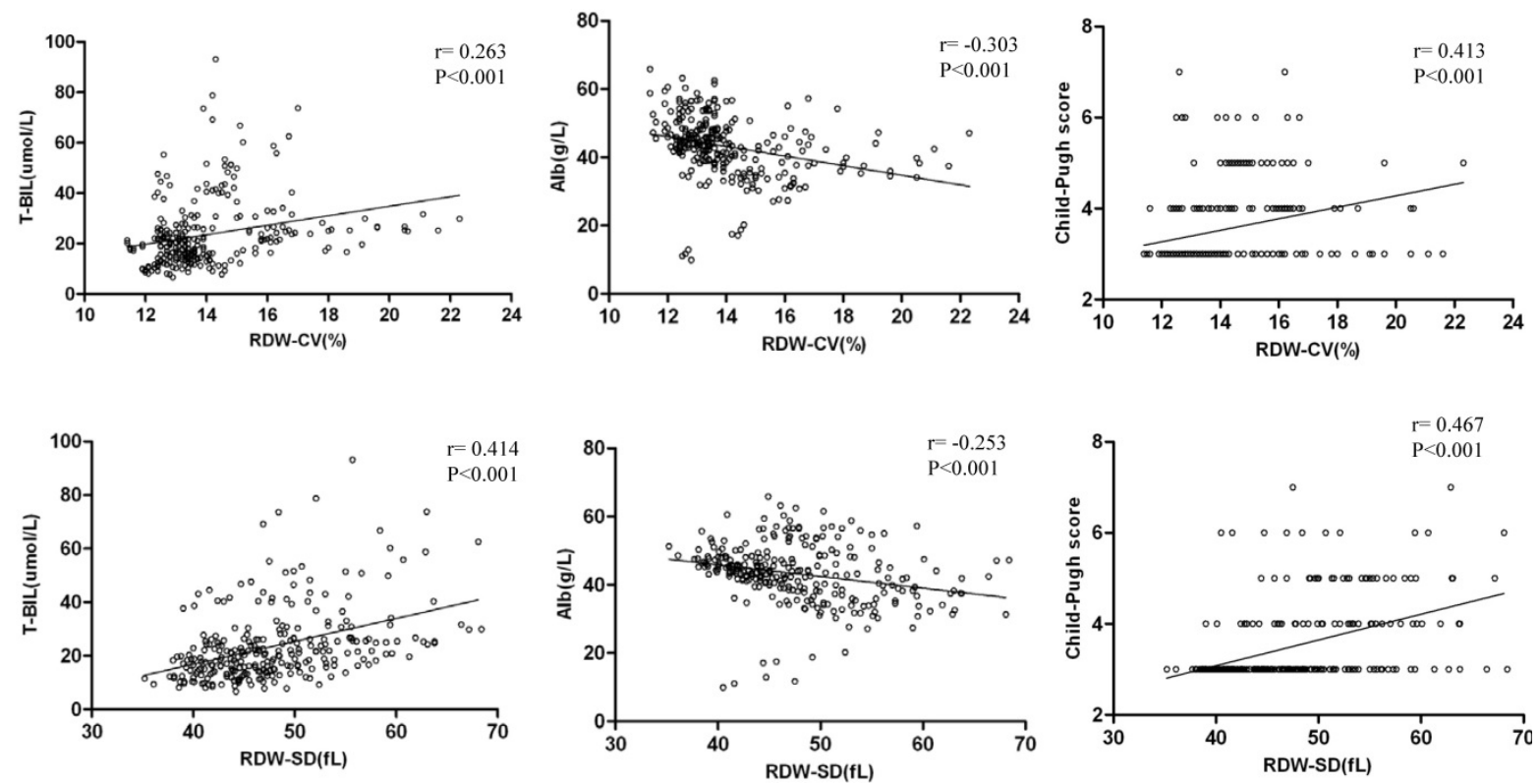

Figure 4. Correlation analysis between RDW levels and clinical indicators of HBV-related hepatocirrhosis patients. Total bilirubin (T-BIL) and albumin levels of each patient with HBV-related hepatocirrhosis were measured and child-Pugh score was calculated. Correlation analysis was conducted between RDW (both CV and SD) and T-BIL, albumin as well as Child-Pugh score of these hepatocirrhosis patients $(n=272)$. Scatter plots with linear fit are shown and $r$ and $P$ values are listed. $P$ values less than 0.05 are regarded as significant.

Table 2. Cut-off value analysis for RDW from ROC curves by the maximum value of Youden index

\begin{tabular}{llllll}
\hline Control group & Patient group & RDW & Cut-off value & Sensitivity \% & Specificity \% \\
\hline CHB and inactive carriers & cirrhosis & SD (fl) & 43.75 & 70.96 & 63.14 \\
& & CV (\%) & 13.05 & 66.91 & 64.69 \\
CHB & cirrhosis & SD (fl) & 43.75 & 70.96 & 58.68 \\
& & CV (\%) & 13.05 & 66.91 & 59.38 \\
inactive carriers & cirrhosis & SD (fl) & 44.05 & 68.38 & 69.71 \\
& & CV (\%) & 13.25 & 59.93 & 87.00 \\
\hline
\end{tabular}


control group:

CHB

Patient group:

RDW-CV

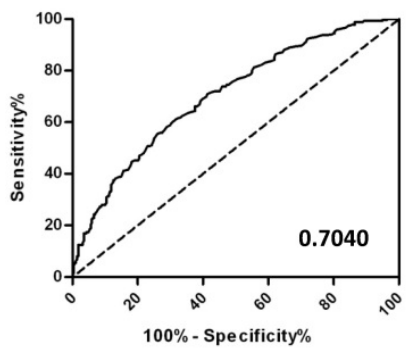

hepatocirrhosis

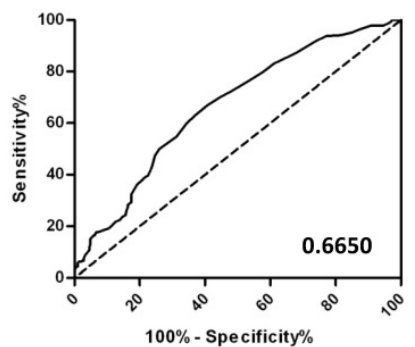

inactive carriers

hepatocirrhosis
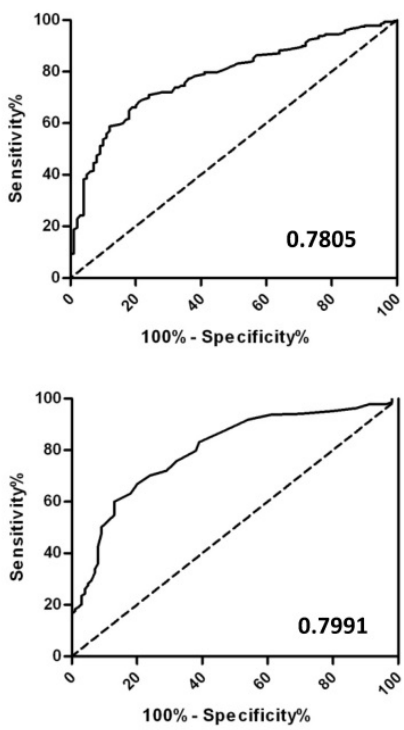

both $\mathrm{CHB}$ and

inactive carriers
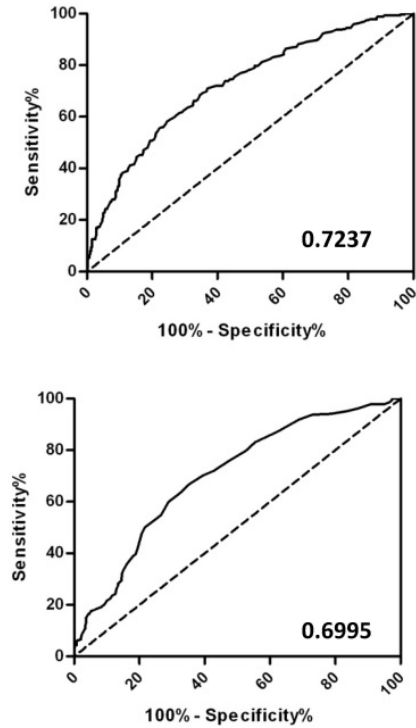

Figure 5. ROC analysis of RDW to distinguish hepatocirrhosis from CHB and inactive HBV carriers. ROC curves were used to evaluate the usefulness of RDW to distinguish hepatocirrhosis from CHB or/and Hepatitis B carriers. The control group included CHB patients (left), inactive HBV carriers (middle), or both (right), and the patient group included patients with HBV-related hepatocirrhosis. Area under curves (AUC) of RDW-SD (up) or RDW-CV (bottom) were calculated and listed.

\section{Discussion}

RDW is a routine laboratory parameter of complete blood count and the cost of measuring RDW is low enough to be extensively used. For a long time, RDW is used for differential diagnosis of anemias in laboratory hematology [1-2]. In recent years, however, a series of reports had been published which suggested RDW was a prognostic marker in a variety of disorders [3-10]. Increased RDW reflects a serious deregulation of erythrocyte homeostasis including both impaired erythropoiesis and abnormal red blood cell survival. The reason of increased RDW may be a variety of underlying metabolic abnormalities, e.g., oxidative stress, inflammation, hypertension, malnutrition, dyslipidemia, and erythrocyte disorders [31].

A few literatures had reported the significance of RDW in HBV-related diseases [18-22]. Until now, however, few researches had studied the dynamic changes of RDW during the process of chronic HBV infection. The clinical significance of RDW in inactive HBV carriers, chronic hepatitis B patients and HBV related hepatocirrhosis remains unclear. In this study, we found that RDW was significantly higher in HBV-related hepatocirrhosis patients and HBeAg+ $\mathrm{CHB}$ patients. Besides, RDW was found to be significantly correlated with biochemical indicators of HBV related hepatocirrhosis patients and could reflect severity of hepatocirrhosis. In addition, RDW could help to diagnose hepatocirrhosis from $\mathrm{CHB}$ and inactive HBV carriers.

HBeAg-positive CHB represents a status of loss of immune tolerance, which lead to necroinflammatory liver injury and the disease can advance to significant fibrosis [32]. For $\mathrm{HBeAg}$ negative $\mathrm{CHB}$, there is $\mathrm{HBV}$ infection, but the histological signs of hepatitis disappear, and serum HBV DNA levels are persistently low [31]. According to our results, $\mathrm{HBeAg}$ positive $\mathrm{CHB}$ patients showed significantly higher RDW than HBeAg negative CHB patients. But there were no significant correlations between RDW and clinical indicators for CHB patients or inactive HBV carriers. The detailed mechanism for higher RDW among HBeAg positive CHB patients should to be considered in depth in future. In addition, we analyzed part of $\mathrm{HBeAg}+\mathrm{CHB}$ patients enrolled in this study, and after antiviral therapy, RDW levels were significantly decreased for patients that underwent HBeAg seroconversion. For patients that are still $\mathrm{HBeAg}+$ after treatment, RDW-SD and RDW-CV were not significantly changed before and after treatment. This part of the results suggests that RDW is associated with the outcome of $\mathrm{CHB}$ after therapy, which could be of clinical significance for evaluating prognosis after CHB treatment.

Whether the change in RDW level is one of the factors leading to disease or the disease causes the 
change of RDW is worthy of further research, and several biological and metabolic abnormalities associated with hepatitis may also have a considerable impact on erythropoiesis. Some pro-inflammatory cytokines were reported to inhibit the synthesis of erythropoietin, impair iron metabolism, thereby impairing red blood cell maturation, leading to immature red blood cells entering the bloodstream, while chronic HBV infection causes obvious inflammatory reactions in the body, so patients The inflammatory response may be responsible for the significant increase in RDW levels in patients with $\mathrm{CHB}$ or cirrhosis that develop from CHB [33-34]. Whether these pro-inflammatory cytokines produce RDW-lowering effects in HBV-associated chronic diseases needs to be validated in future experiments, such as the possibility of placing normal RBCs in sera of different severity of cirrhosis or $\mathrm{CHB}$ patients by in vitro experiments. Systematic studies can also be performed in animal models of $\mathrm{HBV}$ infection. Besides, It is worth noting that portal hypertension in patients with cirrhosis has been reported to cause an increase in plasma volume and may eventually lead to a decrease in RBC survival, which could also be the cause of increased RDW in HBV-related cirrhosis [35] .

As we have known, chronic HBV infection is associated with a wide range of clinical manifestations, from an asymptomatic carrier status with a normal liver histology to severe and chronic liver diseases. Considerable proportion of chronically infected people may develop to cirrhosis, whereas the remainders become inactive carriers. Besides, $0.7 \%$ of asymptomatic HBV carriers could also develop into cirrhosis annually [12-17]. Distinguishing $\mathrm{HBV}$-related hepatocirrhosis from $\mathrm{CHB}$ and inactive carriers is of important clinical significance. According to our results, RDW was significantly increased in cirrhosis patients than in inactive carriers and $\mathrm{CHB}$ patients, which suggest that the dynamic differences of RDW levels may help their differential diagnosis. Our preliminary receiver operating characteristics (ROC) curve analysis proved that RDW was one potential indicator that could help to diagnose hepatocirrhosis from other HBV-related chronic diseases. In addition, RDW was correlated with T-BIL albumin and child-Pugh scores, which suggested that increased RDW was associated with conditions of hepatocirrhosis patients.

There are limitations in this research and still much more work needs to be done about the significance of increased RDW levels in HBV-related chronic diseases. The sample size needs to be enlarged in the future and the critical cut-off value of RDW to distinguish hepatocirrhosis patients from $\mathrm{CHB}$ patients or carriers needs to be determined according to multi-centered research as well. Generally, in this study, we inquired into the change of RDW levels in HBV-related chronic diseases and studied the clinical significance of RDW, which could contribute to a comprehensive understanding of the clinical significance of RDW during HBV infection.

\section{Abbreviations}

Alb: albumin; ALT: alanine aminotransferase; AST: aspartate aminotransferase; AUC: area under curves; CHB: chronic hepatitis B; CMIA: chemiluminescent micro-particle immunoassay; $\mathrm{CV}$ : coefficient of variation; $\mathrm{HBcAb}$ : hepatitis $\mathrm{B}$ core antibody; HBeAb: hepatitis B e antibody; HBeAg: hepatitis B e antigen; HBsAb: hepatitis B surface antibody; HBsAg: hepatitis B surface antigen; HBV: Hepatitis B Virus; HCC: hepatocellular carcinoma; RPR: RDW to Platelet Ratio; RDW: Red cell distribution width; ROC: Receiver operating characteristics; SD: standard deviation; T-BIL: total bilirubin.

\section{Supplementary Material}

Supplementary tables.

http://www.medsci.org/v16p0720s1.pdf

\section{Acknowledgment}

This work was supported by grants from Natural Science Foundation of China (81401298, 81871230), the Beijing Natural Science Foundation (7163228) and Peking University People's Hospital Scientific Research development Funds(RS2018-01). We thank Department of Hepatology of Peking University People's Hospital for the share of medical records.

\section{Competing Interests}

The authors have declared that no competing interest exists.

\section{References}

1. Aulakh R, Sohi I, Singh T, et al. Red cell distribution width (RDW) in the diagnosis of iron deficiency with microcytic hypochromic anemia. Indian J Pediatr. 2009; 76: 265-8.

2. Aslan D, Gumruk F, Gurgey A, Altay C. Importance of RDW value in differential diagnosis of hypochrome anemias. Am J Hematol. 2002; 69:31-3.

3. Arbel Y, Weitzman D, Raz R, et al. Red blood cell distribution width and the risk of cardiovascular morbidity and all-cause mortality. Thromb Haemostasis. 2014; 111: 300-7.

4. $\mathrm{Hu} \mathrm{L}, \mathrm{Li}$ M, Ding Y, et al. Prognostic value of RDW in cancers: a systematic review and meta-analysis. Oncotarget. 2017; 7: 67020-32.

5. Yazici P, Demir U, Bozkurt E, Isil GR., Mihmanli M. The role of red cell distribution width in the prognosis of patients with gastric cancer. Cancer Biomark. 2017; 18: 19-25.

6. Koma Y, Onishi A, Matsuoka H, et al. Increased red blood cell distribution width associates with cancer stage and prognosis in patients with lung cancer. PloS One. 2013; 8:e80240.

7. Engstrom G, Smith JG, Persson M, Nilsson PM, Melander O, Hedblad B. Red cell distribution width, haemoglobin A1c and incidence of diabetes mellitus. J Intern Med. 2014; 276:174-83. 
8. Lippi G, Targher G, Salvagno GL, Guidi GC. Increased red blood cell distribution width (RDW) is associated with higher glycosylated hemoglobin (HbA1c) in the elderly. Clin Lab. 2014; 60: 2095-8.

9. Seyhan E C, Ozgul MA, Tutar N, Omur I M, Uysal A, Altın S. Red blood cell distribution and survival in patients with chronic obstructive pulmonary disease. COPD. 2013; 10: 416-24.

10. Lippi G, Targher G, Montagnana M, Salvagno GL, Zoppini G, Guidi GC Relationship between red blood cell distribution width and kidney function tests in a large cohort of unselected outpatients. Scand J Clin Lab Inv. 2008; 68: 745-8.

11. Beaven SW. No Nukes: A New Way to Treat Hepatitis B. Sci Trans Med. 2014; 229: ec52.

12. Lu FM, Zhuang H. Management of hepatitis B in China. Chinese Med J. 2009; 122: 3-4

13. Liaw Y F, Chu CM. Hepatitis B virus infection. Lancet. 2009; 373: 582-92.

14. European Association For The Study Of The Liver. EASL 2017 Clinical Practice Guidelines on the management of hepatitis B virus infection. J Hepatol. 2017;67:370-98.

15. Lok AS. Chronic hepatitis B. N Engl J Med. 2002; 346:1682-3.

16. Hsu YS, Chien RN, Yeh CT, et al. Long-term outcome after spontaneous HBeAg seroconversion in patients with chronic hepatitis B. Hepatology. 2002;35: 1522-7.

17. Wu JC, Hwang S J, Lai CR, et al. Factors predictive of liver cirrhosis in patients with chronic hepatitis B: a multivariate analysis in a longitudinal study. Eur J Gastroen Hepat. 2000; 12: 687-93.

18. Yu MW, Hsu FC, Sheen I, et al. Prospective study of hepatocellular carcinoma and liver cirrhosis in asymptomatic chronic hepatitis B virus carriers. Am J Epidemiol. 1997; 145: 1039-47.

19. Lou Y, Wang M, Mao W. Clinical usefulness of measuring red blood cell distribution width in patients with hepatitis B. PloS One. 2012;7: e37644.

20. Huang R, Yang $\mathrm{C}$, Wu K, et al. Red cell distribution width as a potential index to assess the severity of hepatitis B virus-related liver diseases. Hepatol Res. 2014; 44:e464-70

21. Xu WS, Oiu XM, Ou OS, et al. Red blood cell distribution width levels correlate with liver fibrosis and inflammation: a noninvasive serum marker panel to predict the severity of fibrosis and inflammation in patients with hepatitis B. Medicine. 2015; 94: e612.

22. Karagoz E, Ulcay A, Tanoglu A, et al. Clinical usefulness of mean platelet volume and red blood cell distribution width to platelet ratio for predicting the severity of hepatic fibrosis in chronic hepatitis B virus patients. Eur J Gastroen Hepat. 2014; 26: 1320-4.

23. Chen B, Ye B, Zhang J, Ying L, Chen Y. RDW to platelet ratio: a novel noninvasive index for predicting hepatic fibrosis and cirrhosis in chronic hepatitis B. PLos One. 2013; 8: e68780.

24. Fan X, Deng H, Wang X, et al. Association of red blood cell distribution width with severity of hepatitis B virus-related liver diseases. Clinica Chimica Acta. 2018; 482:155-60

25. Cholongitas E, Papatheodoridis GV, Vangeli $M$, Terreni $N$, Patch $D$, Burroughs AK. Systematic review: the model for end-stage liver disease-should it replace Child-Pugh's classification for assessing prognosis in cirrhosis? Aliment Pharm Ther. 2005; 22:1079-89.

26. Karasu Z, Tekin F, Ersoz G, et al. Liver fibrosis is associated with decreased peripheral platelet count in patients with chronic hepatitis B and C. Digest Dis Sci. 2007;52: 1535-9.

27. Pradella $\mathrm{P}$, Bonetto $\mathrm{S}$, Turchetto $\mathrm{S}$, et al. Platelet production and destruction in liver cirrhosis. J Hepatol. 2011; 54: 894-900.

28. Kuriya SI, Nomura T. A study on the mechanism of anemia and leukopenia in liver cirrhosis. Jpn J Med. 1988; 27:155-9.

29. Martinot-Peignoux M, Carvalho-Filho R, Lapalus M, et al. Hepatitis B surface antigen serum level is associated with fibrosis severity in treatment-naive, $\mathrm{e}$ antigen-positive patients. J Hepatol. 2013; 58: 1089-95.

30. Ikeda K, Saitoh S, Koida I, et al. A multivariate analysis of risk factors for hepatocellular carcinogenesis: a prospective observation of 795 patients with viral and alcoholic cirrhosis. Hepatology. 1993;18: 47-53.

31. Salvagno GL, Sanchis-Gomar F, Picanza A, Lippi G. Red blood cell distribution width: a simple parameter with multiple clinical applications. Crit Rev Cl Lab Sci. 2015; 52: 86-105

32. Hadziyannis SJ, Vassilopoulos D. Hepatitis B e antigen-negative chronic hepatitis B. Hepatology. 2001; 34: 617-24.

33. Jelkmann W. Proinflammatory cytokines lowering erythropoietin production. J Interferon Cytokine Res. 1998; 18:555-9.

34. Weiss G, Goodnough LT. Anemia of chronic disease. N Engl J Med. 2005;352:1011-23.

35. Kimber C, Deller DJ, Ibbotson RN, Lander H. The mechanism of anaemia in chronic liver disease. Q J Med 1965:34:33-64.

\section{Author Biography}

Dr. Chen Liu is an associate professor at Peking University People's Hospital, Beijing, China. He has published 12 publications including Arthritis \& Rheumatology, Journal of immunology, leukemia research etc. The current research interests in Professor Liu's group include: (1) Clinical Significance of coagulation and hematology indicators in diseases. (2) Development and clinical significance of regulatory $\mathrm{T}$ cells. 